\title{
COMPORTEMENT CRITIQUE DE LA VITESSE ET DE LATTÉNUATION DES ULTRASONS AU VOISINAGE D'UNE TRANSITION SMECTIQUE A - SMECTIQUE C
}

\author{
D. COLLIN, J.L. GALLANI et P. MARTINOTY
}

Laboratoire d'Ultrasons et de Dynamique des Fluides Complexes, Unité de Recherche Associée au C.N.R.S. $N^{\circ} 851$, Université Louis Pasteur, 4 rue Blaise Pascal, F-67070 Strasbourg cedex, France

\begin{abstract}
.
We present measurements of the damping and velocity of ultrasound, performed near the Smectic A - smectic C phase transition of. TBBA (terephthal-bis-p-p'-butylaniline), showing a critical behavior close to that expected for the 3DXY universality class, whereas the specific heat data suggest that this transition is of the Landau-type (no fluctuations). This apparent contradiction is explained by showing that the Ginzburg criterion depends on the observable under study.
\end{abstract}

Les phases Smectique A et Smectique C des cristaux liquides sont constituées de couches fluides, équidistantes, empilées les unes sur les autres. Dans la phase Smectique A les molécules sont parallèles à la normale aux couches alors que dans la phase Smectique $C$ elles sont inclinées d'un angle $\varphi$ par rapport à cette normale.

La transition Smectique A - Smectique $C$ a fait l'objet ces dernières années d'un grand nombre de travaux expérimentaux qui ont été motivés par les approches théoriques reposant sur la reconnaissance de l'universalité des phénomènes critiques. La plupart des résultats obtenus, notamment ceux de chaleur spécifique, suggèrent cependant que cette transition est de type Landau (pas de fluctuations) [1] alors qu'il était prévu (de Gennes) [2] qu'elle appartienne à la même classe d'universalité (3DXY) que la transition $\lambda$ de $1^{\prime}$ hélium.

Nous présentons ici des mesures de l'atténuation $\alpha$ et de la vitesse $V$ des ultrasons effectuées dans le terephthal-bis-p-p'-butylaniline (TBBA). Les échantillons ont été orientés à l'aide d'un champ magnétique de $10 \mathrm{kG}$ et les mesures ultrasonores faites en fonction de $1^{\prime}$ angle $\theta$ entre la direction de propagation du son et la normale aux couches (1a réf. 3 donne une description détaillée du dispositif de mesure et de la préparation des échantillons). Ces mesures mettent en évidence des effets de fluctuations importants, encore visibles $10^{\circ} \mathrm{C}$ au-dessus de la transition comme le montrent les figures 1 et 2 relatives à $l^{\prime}$ atténuation et à $1 a$ vitesse. Ces effets sont tout à fait inattendus dans la mesure où ils $n^{\prime}$ apparaissent pas sur les mesures de chaleur spécifique effectuées sur le même composé [4] (fig. 3), et prouvent que le modèle de Landau ne s'applique pas à la transition. Les figures 1 et 2 montrent également une anisotropie marquée des effets prétransitionnels qui provient du fait que 1'interaction entre 1'onde ultrasonore et 1'orientation des molécules se réalise via une modulation de la distance intercouche [5]. I'intensité de cette modulation dépend de $I^{\prime}$ angle $\theta$; elle est maximale pour $\theta=0^{\circ}$.

$L^{\prime}$ analyse des mesures d'atténuation a été effectuée pour $\theta=45^{\circ}$, car 1 es effets anharmoniques, caractéristiques des phases smectiques, ne contribuent pas à 1'atténuation pour cette orientation [6]. La fig. 4 présente la variation de la partie critique de $l^{\prime}$ atténuation en fonction de $T-T_{A C}$ pour différentes fréquences. 

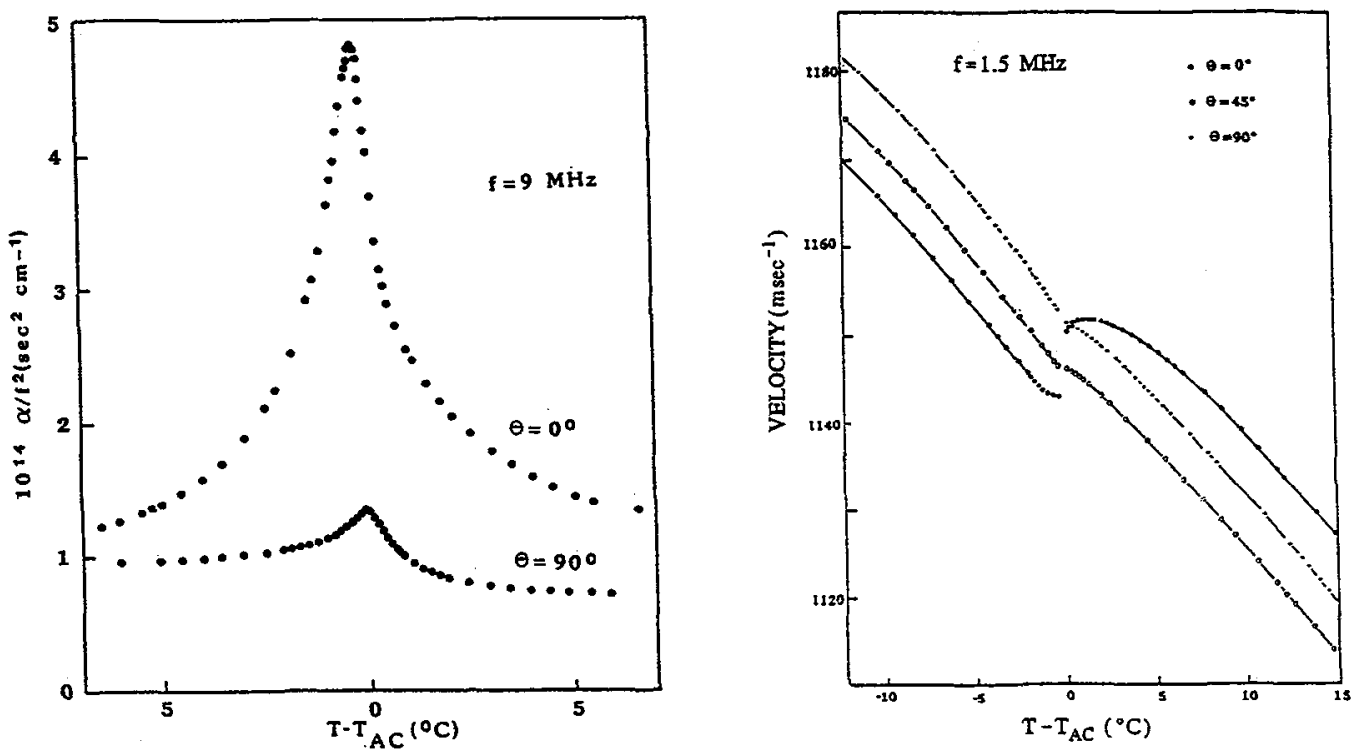

Figs. 1 et 2 . Comportement de l'atténuation et de la vitesse ultrasonores au voisinage de la transition $\mathrm{SmA}-\mathrm{SmC}$ montrant $\mathbf{l}^{\prime}$ existence d'effets prétransitionnels marqués et anisotropes.

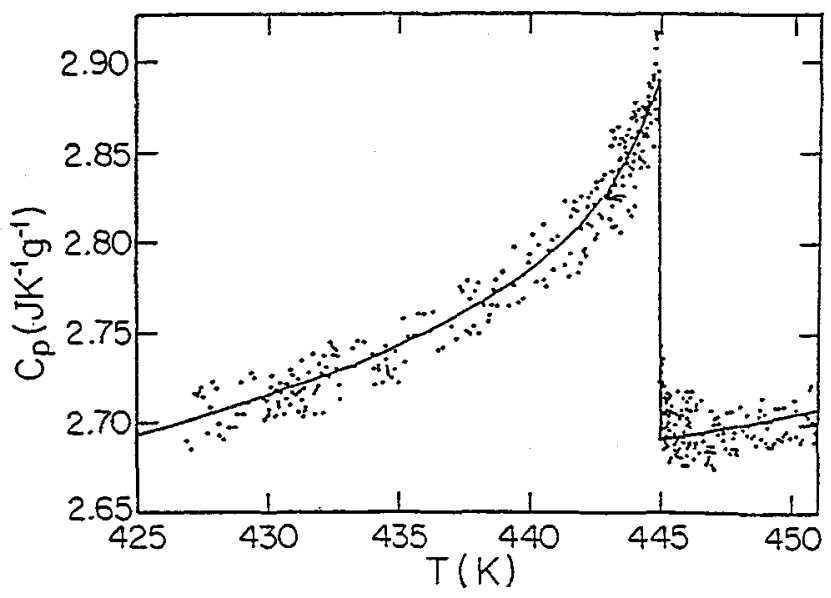

Fig. 3. Comportement thermique de la chaleur spécifique. La ligne en trait plein représente la courbe théorique calculée avec le modèle de Landau ( $d^{\prime}$ après la réf. 4). 
Ces données ont été analysées à $I^{\prime}$ aide de la loi d'échelle suivante déjà utilisée pour l'hélium :

$$
\alpha / \omega^{2}=\frac{A_{0} r t^{-\bar{\alpha}}}{C+(\omega \tau)^{-1-\bar{\alpha} / 2 \nu}}
$$

où $A_{0}$ et $C$ sont des constantes, $t$ l'écart à la température de transittion $\left(t=\mathrm{T}-\mathrm{T}_{\mathrm{AC}}\right)$ et $\tau$ le temps de relaxation du paramètre d'ordre $\left(\tau=\tau_{0} t^{-z \nu}\right)$. Ces analyses donnent $-0.02<\bar{\alpha}<0.02$ et $1.18<z \nu<1.33$. Ces valeurs sont très voisines de celles attendues pour la classe d'universalité $3 \mathrm{DXY}(\bar{\alpha}=-0.02$, $z \nu=1.32$ ). II $n^{\prime}$ est toutefois pas possible de savoir si nos mesures correspondent précisément au régime asymptotique (comportement 3DXY), ou si elles se situent dans un régime préasymptotique caractérisé par une divergence de la chaleur spécifique liée à une valeur légèrement positive de l'exposant $\bar{\alpha}$.

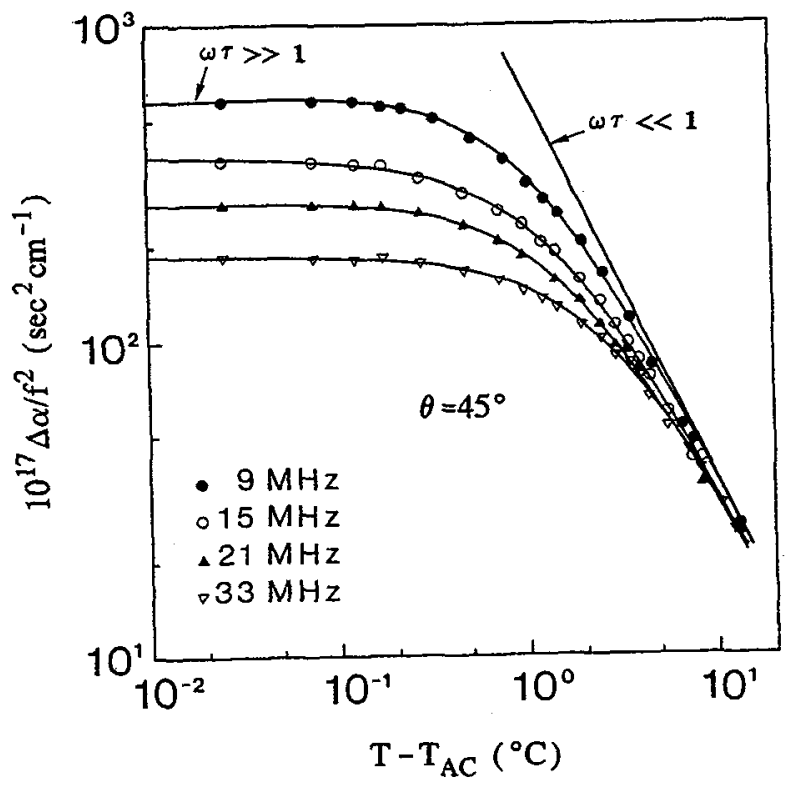

Fig. 4. Comportement critique de $I^{\prime}$ atténuation. Les lignes en trait plein représentent les différents ajustements réalisés avec la formule (1).

La contradiction apparente entre le comportement de la chaleur spécifique (de type Landau) et celui de la vitesse (de type crítique) peut être expliquée en montrant que la largeur $\Delta \mathrm{T}_{c}$ de la région critique, qui est donnée par le critère de Ginzburg, dépend de l'observable considérée [7]. Ceci provient du fait que le comportement de la vitesse doit être déduit de l'ênergie interne F (propagation adiabatique) alors que celui de la chaleur spécifique doit être déduit de l'énergie libre de Gibbs. Le calcul montre que 


$$
\left(\Delta \mathrm{T}_{c}\right)_{\text {élastique }} /\left(\Delta \mathrm{T}_{\mathrm{c}}\right)_{c p}=\left(\frac{\mathrm{b}}{\mathrm{b}^{*}}\right)^{2}
$$

où $b$ et $b^{*}$ sont respectivement les coefficients des termes de degré 4 dans les développements de Landau de $F$ et $G$. b et $b^{*}$ sont reliés par la relation

$$
b^{*}=b\left[1+\frac{\Delta\left(A B-C^{2}\right)}{A B-C^{2}}\right]
$$

dans laquelle $A, B$ et $C$ sont les constantes élastiques associées à la vitesse $\left(\rho V^{2}(\theta)=A-2 C \cos ^{2} \theta+B \cos ^{4} \theta\right)$. La quantité $\Delta\left(A B-C^{2}\right)$ désigne le saut de $A B-C^{2}$ à la transition. Les constantes $A B$ et $C$ et leur variation thermique peuvent etre déduites des mesures de la fig.2. Il est donc possible de déterminer expérimentalement le rapport $b / b^{*}$. On trouve $b / b^{*} \simeq 10$, ce qui entraine que

$$
\left(\Delta \mathrm{T}_{\mathrm{c}}\right)_{\text {élastique }} \approx 100\left(\Delta \mathrm{T}_{\mathrm{c}}\right)_{\mathrm{cp}}
$$

Il en résulte que si la largeur de la région critique associée à la vitesse est de $1^{\prime}$ ordre de $10^{\circ} \mathrm{C}$, comme le suggère la fig. 2, celle associée à la chaleur spécifique est inférieure à $0.1^{\circ} \mathrm{C}$. Cette différence explique que les mesures de vitesse présentent un comportement critique, contrairement aux mesures de chaleur spécifique qui présentent un comportement de type Landau.

Le fait que la région critique soit atteinte plus tôt dans le cas des constantes élastiques (ou de la vitesse) que dans le cas de la chaleur spécifique semble être un phénomène général, comme le suggèrent des résultats obtenus aux transitions ferroélectriques [8], mais qui n'a pas été reconnu jusqu'à présent.

[1] Voir par exemple BIRGENEAU, R, J, GARLAND, C, W, KORTAN, A, R, LITSTER, J, D, MEICHLE, M, OCKO, B, M, ROSENBLATT, $C, Y U, L, J$, and GOODBY, I, Phys. Rev. A. 27 (1983) 1251.

[2] DE GENNES, P,G, G.R.Acad.Sci. Paris 274 (1972) 758 B.

[3] COLLIN, D, GALIANI, $J ; L$, and MARTINOTY, P, Phys. Rev. A. 34 (1986) 2255. Ibidem Phys. Rev. Lett. 58 (1987) 254.

[4] DAS, P, EMA, K, and GARLAND, C,W, Liq. Cryst. 4 (1989) 205.

[5] ANDERECK, B, S, and SWIFT, J, Phys. Rev. A 25 (1982) 1084.

[6] Pour une étude des effets anharmoniques voir la réf.3.

[7] BENGUIGUI, L, and MARTINOTY, P, Phys. Rev. Lett. 63 (1989) 774.

[8] Pour les constantes élastiques voir YAO, $\mathrm{W}$, CUMMINS, $\mathrm{H}, \mathrm{Z}$, and BRUCE, $\mathrm{R}, \mathrm{H}$, Phys. Rev. B 24 (1981) 424 ; pour la chaleur spécifique voir FOUSKOVA, A, J. Phys. Soc. Jpn 27 (1969) 1699. 